\title{
Pericardial Closure Defect with Right Atrial Herniation in a Dog
}

\author{
Radu Andrei BAISAN ${ }^{1 *}$, Magdalena Cristina BARBAZAN ${ }^{1}$, Diego PIANTEDOSI ${ }^{2}$, Vasile VULPE ${ }^{1}$ \\ ${ }^{1}$ Department of Clinical Sciences, Faculty of Veterinary Medicine, USAMV Iasi, Romania. \\ ${ }^{2}$ Department of Veterinary Medicine and Animal Productions, Univestity of Naples Federico II, Italy \\ *Corresponding author: baisan.andrei_mv@yahoo.com
}

Bulletin UASVM Veterinary Medicine 72(2) / 2015,

Print ISSN 1843-5270; Electronic ISSN 1843-5378

DOI:10.15835/buasvmcn-vm: 11400

\begin{abstract}
A 16 years old German Shepherd dog was referred to the teaching hospital of Veterinary Medicine Faculty of Iasi, with cardio-respiratory symptoms. Clinical examination, electrocardiography, thoracic X-ray and echocardiography have been performed and congestive heart failure due to right cardiac dilatation was diagnosed. Due to the dog's death, necropsy have been performed and revealed a right pericardial closure defect with the hernia and incarceration of the right atrium in the mediastinum. The aim of the study is to describe an uncommon cardiac pathology in a geriatric dog represented by a pericardial tear with the right auricular hernia and incarceration and to raise hypothesis of the pathological findings etiology.
\end{abstract}

Keywords: atrial hernia, dog, heart

\section{INTRODUCTION}

The pericardium is a fibroserous membrane continued with the sterno-pericardial ligament attached to the ventral aspect of the diaphragm, anchoring the heart in the thorax. Also, the pericardium restrains the cardiac filling and protects the heart from dilatation and myocardial rupture (Sisson and Thomas, 1999). Partial pericardial defects are rare in human medicine, usually silent anomalies, left sided and congenital origin, approximated to $1 / 10.000$ and most, incidental findings, engendering a small risk of cardiac hernia (Ilhan et al., 2012; Vesely and Julsrud, 1989; Yousefzadeh et al., 1982). Hernia of the left and right atrium, atrium and ventricle, cardiac apex, or entire heart, have been reported in humans (Sisson and Thomas, 1999) and dogs (Gaag and Luer, 1977). The right pericardial anomalies are very rare findings and few cases have been described even in human medicine (Minocha et al., 1979). Sometimes, along with hernia, a myocardial strangulation is produced, generating blood ceasing and clotting. Depending on the size and location of the hernia, other topographic or physiological changes may be found. The pericardial defect can be congenital or acquired. The factors responsible for the congenital closure defects of the pericardium are connected to the embryogenesis of the coelom (Gaag and Luer, 1977). Acquired pericardial defects are often found in adults and may result due to pericardial injuries such as trauma, pericarditis, tamponade, or disruption of the pericardium (Liedtke and DeMuth, 1973). The aim of the study is to describe an uncommon cardiac pathology in a geriatric dog represented by a pericardial tear with right auricular hernia and incarceration and to explain the pathological mechanism comparing to human medicine findings, considering the association of two uncommon pathologies. The peculiarity of this case is represented by the spectacular shape and dimensions of the right auricle herniated through the pericardial tear and the unknown etiology of the pathological findings. 


\section{MATERIALS AND METHODS}

A 16 years old male German Shepherd dog, with the right forelimb amputated, was referred to the teaching hospital of Veterinary Medicine Faculty from Iasi with torpidity, severe dyspnea and respiratory wheezing. No medical history was available because it was a stray dog. Clinical examination has been performed and further special exams have been recommended. Thoracic $\mathrm{X}$-ray in lateral and sternal recumbency, cardiac ultrasonography in right lateral recumbency according to ACVIM recommendations standards for transthoracic echocardiography (Thomas et al., 1993) and 5 minutes, six-lead electrocardiography have been performed. The clinical status of the dog worsened very fast and the dog died. Furthermore, necropsy was performed.

\section{RESULTS AND DISCUSSION}

Clinical examination showed cyanosis, severe respiratory restrain, wheezing and cardiac thrill. Auscultation revealed tachycardia and delirium cordis sound. Electrocardiography revealed normal electrical axis, supraventricular rhythm with tachyarrhythmia (140 bpm), tall $\mathrm{R}$ waves and atrial fibrilation. Lateral X-ray revealed dilatation of the right ventricle and the presence of a detached mass on the cranial silhouette of the heart. Cardiac silhouette extended over five intercostals spaces and the vertebral heart score (Buchanan and Bucheler, 1995) was 13. Caudal vena cava was enlarged and oblique due to cardiac cranial rotation. The trachea was dorsally displaced, the interstitial and vascular patterns were highlighted and the tracheal lymph node was enlarged. Spondylosis between vertebrae T4-T6 was present (Fig. 1). The sternal recumbency X-ray showed enlarged cranial-caudal cardiac silhouette, extended over 7,5 intercostal spaces being entirely displaced in the left side of the thorax. No signs of rib fracture were found.

The ultrasonographic examination showed an enlarged left atrium, with the ratio La/Ao of 2.21. The mitral valve leaflets were thickened. The dog died shortly after the investigations. Necropsy examination revealed the heart entirely displaced in the left side of the thoracic cavity. A round orifice in the pericardium surrounding the herniated right atrium was visible, the cranial and caudal vena cava were visible enlarged, but the phrenic nerve was intact. The herniated right atrium was orientated towards the apex (Fig. 2). No adhesions between the herniated auricle and other structures were found. The pericardial tear margins were thickened and regular, oval shaped. The heart dissection showed a red, smooth, gelatinous, unattached, post-mortem, massive clot inside the right auricle and the atrium wall was thinned and stretched. No adult heartworms were found in the right ventricle or the main pulmonary arteries.

In this case, it could not be specified for how long the hernia was present, nor when the pericardial defect did appear. However, it seems that the pericardial defect was old because of the fibrous margins of the orifice and the dimensions of the herniated auricle. There have been information that the dog suffered a car accident in the past, resulting in the right forelimb amputation. In human medicine literature, this pathology is well described. A congenital right pericardial defect and right atrial appendage hernia with the luxation of the heart to the left side have been reported in an eight month old boy (Karakurt et al., 2006). On the other side, another report described a right atrial hernia thruogh a vertical right pericardial tear, three months after a chest trauma, in a fourteen years old boy (Yousefzadeh etal.,1982). Also, a case of traumatic tricuspid regurgitation due to cordae tendineae rupture associated with congenital pericardial defect four years post-trauma has been described (Higashikawa et al., 1997). Our patient did not have ruptured cordae tendineae, but it is possible to associate the findings with the accident the dog suffered in the past. The peculiarity of this case is represented by the great dimension of the herniated and incarcerated atrium, extending towards the apex. Either of presumptions, that the pericardial defect was congenital or acquired, the remodeling of the right atrium wall occurred secondary to pericardial tear. Hernia of the right atrium due to pericardial defect, along with right auricular aneurysm should be included in the differential diagnosis of cranial mediastinal masses (Toaldo et al., 2010). Imaging exams are highly recommended for differential diagnosis. The X-ray of a cranial mediastinum mass would reveal the silhouette of the mass superimposed over the cardiac silhouette, creating a more radiopaque area, or a diffuse radiopaque area, usually without clear outline. In case of right auricular aneurysm, the pericardial silhouette surrounding the heart 
and the aneurysm would be visible, creating a more radiopaque zone containing the heart and the aneurysm. The difference in this case, was that between the cranial aspect of the heart and the caudal aspect of the auricle hernia, a radiolucent zone (pulmonary tissue) was visible (Fig. 1). From author`s knowledge, there are very few cases of pericardial closure defects reported in dogs and the most complete study was published in 1977 reporting eight cases of pericardial defects in dog, from which only one was found to have a single pericardial defect on the right cranial side, also with the partial auricle and ventricle protrusion (Gaag and Luer, 1977).

\section{CONCLUSION}

The case presented is a very rare finding, both in human and veterinary medicine, two uncommon pathologies being associated. Imagistic examination have a great role in the differential

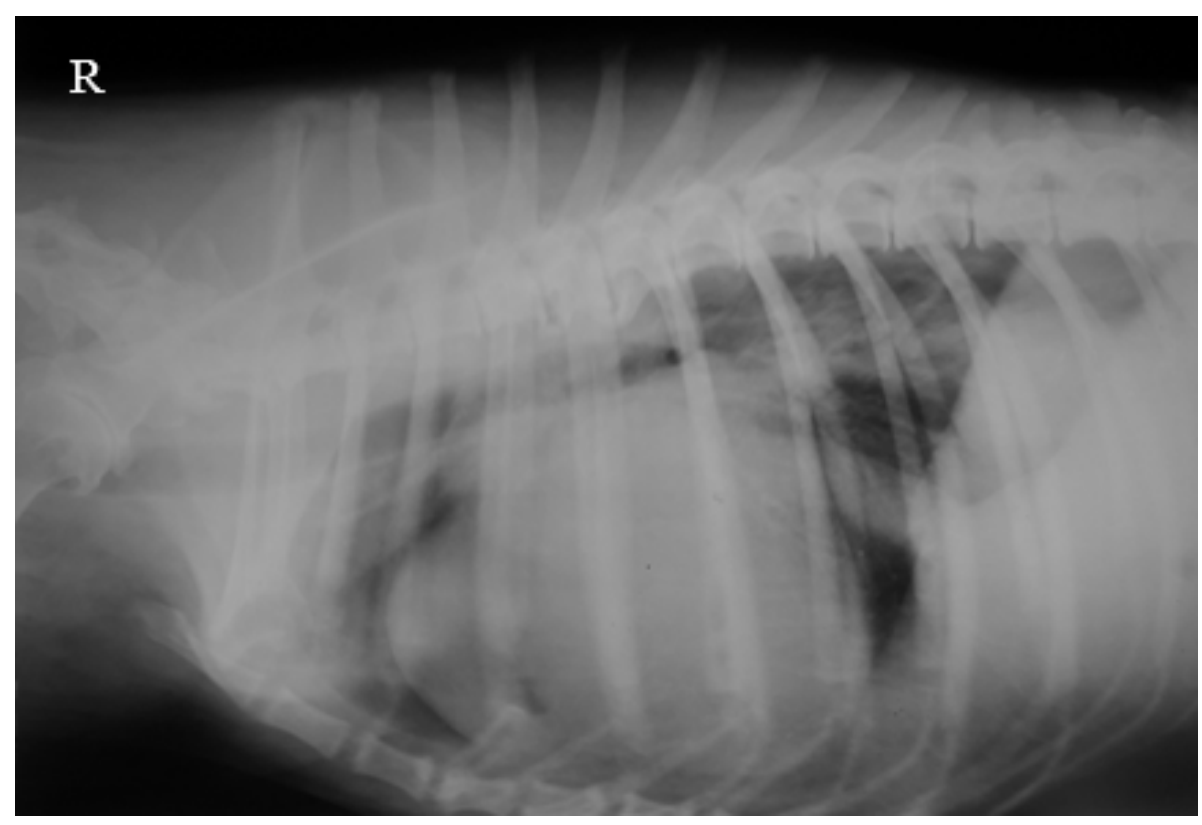

Fig.1. Right lateral radiography of the of the thorax showing modified silhouette of the heart with a cranial radiopaque mass, marked interstitial and vascular pattern and the dorsal displacement of the trachea
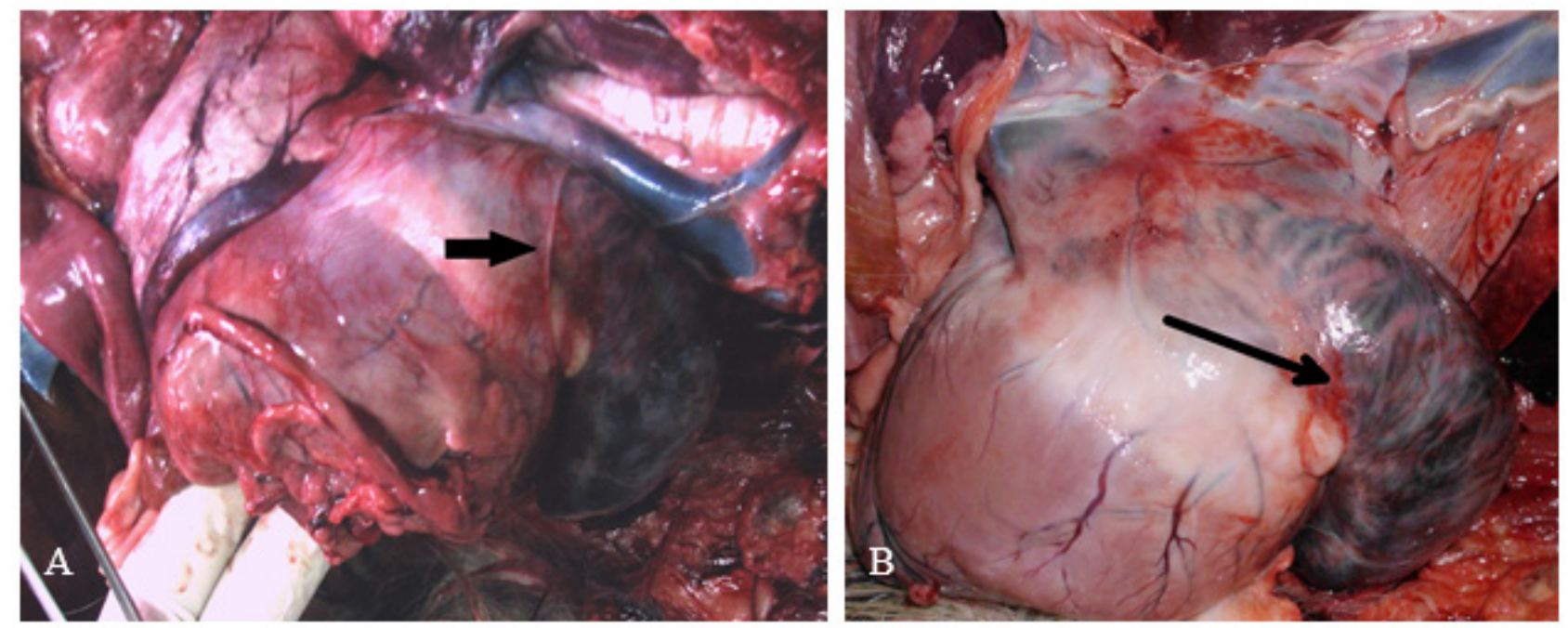

Fig. 2. Gross pathology of the heart; A. short arrow indicating the pericardium margin of the tear; B. long arrow indicating the herniated atrium after the pericardium was removed; 
diagnostic, where hernia of the right atrium due to pericardial defect, along with right auricular aneurysm or cranial mediastinal masses should be included.

\section{REFERENCES}

1. Buchanan JW, Bucheler J (1995). Vertebral Scale System to Measure Canine Heart Size in Radiographs. J Am Vet Med Assoc 206:194-199.

2. Gaag IV, Luer JT (1977). Eight cases of pericardial defects in the dog. Vet Pathol 14:14-18.

3. Higashikawa M, Nishio M, Kanamori T, Nakamura $Y$ Kinoshita M (1997). Traumatic t ricuspid regurgitation associated with congenital partial pericardial defect. Japanese Circulation Journal-English Edition 61:358-360.

4. Ilhan E, Dayi SU, Guvenc TS, Altay S, Dursun M, Hatipsoylu E,Tayyareci G (2012). Congenital absence of the pericardium: a rare cause of right ventricular dilatation and levoposition of the heart. Cardiol J 19:408-411.

5. Karakurt C, Oguz D, Karademir S, Sungur M, Ocal B (2006) Congenital partial pericardial defect and herniated right atrial appendage: a rare anomaly. Echocardiography 23:784-786.
6. Liedtke AJ, DeMuth WE, Jr (1973). Nonpenetrating cardiac injuries: a collective review. Am Heart J 86:687-697.

7. Minocha GK, Falicov RE,Nijensohn E (1979). Partial rightsided congenital pericardial defect with herniation of right atrium and right ventricle. Chest 76:484-486.

8. Sisson D,Thomas WP (1999). Pericardial disease and cardiac tumors. In: Fox PR,Sisson D, Moise NS (Eds). Texbook of canine and feline cardiology, WB Saundres, Philadelphia, 679-702.

9. Thomas WP, Gaber CE, Jacobs GJ, Kaplan PM, Lombard CW, Moise NS, Moses BL (1993). Recommendations for standards in transthoracic two-dimensional echocardiography in the dog and cat. Echocardiography Committee of the Specialty of Cardiology, American College of Veterinary Internal Medicine. J Vet Intern Med 7:247-252.

10. Toaldo MB, Diana A, Morini M, Cipone M (2010). Imaging Diagnosis-Intrapericardia Right Auricle Aneurysm in a Dog. Vet Radiol \& Ultrasound 51:512-515.

11. Vesely T, Julsrud PR (1989). Congenital Absence of the Pericardium and Its Relationship to the Ligamentum Arteriosum. Surg Radiol Anat 11:171-175.

12. Yousefzadeh DK, Mojtahedi S, Marvin WJ, Lu CH, Levine AH (1982). Pericardial defect: unusual presentation, location, and etiology. Pediatr Radiol 12:279-283. 